\title{
Surface Waves along the Martian Ionopause
}

\author{
P. Chmielewski ${ }^{a}$, K. Murawski ${ }^{a}$ And N. KumaR ${ }^{b}$ \\ ${ }^{a}$ Group of Astrophysics, Institute of Physics, UMCS, E. Radziszewskiego 10, 20-033 Lublin, Poland \\ ${ }^{b}$ Department of Mathematics, M.M.H. College, Ghaziabad, UP, India
}

(Received April 8, 2011; in final form May 25, 2012)

\begin{abstract}
We study the behaviour of surface waves propagating along the ionopause of the planet Mars and derive the onset criteria for unstable Kelvin-Helmholtz modes and negative energy waves at the Martian ionopause. Growth rate of unstable Kelvin-Helmholtz mode decreases with wavelength. The backward propagating waves became negative energy waves for the ionosheath velocity higher than critical velocity. It is found that short wavelength surface waves propagating along the Martian ionopause are less dispersive than long waves.
\end{abstract}

DOI: 10.12693/APhysPolA.123.156

PACS: 94.20.wh, 96.30.Gc

\section{Introduction}

The first observation of the planet Mars by means of a primitive telescope was made by Galileo Galilei in 1610 [1]. With gradual improvement of spatial resolution of telescopes, more details about motion and appearance of the planet were reported subsequently. Among others, the south pole was perceived first by Huyghens in 1672 and two Martian satellites were discovered in August 1877 by Asaph Hall. A map of the surface of Mars was sketched the same year by Nathaniel Green.

The second half of the twentieth century brought new possibilities in situ observations of the planet via spacecrafts. In the late fifties, the satellites Luna- 1 and Luna-2 discovered the solar wind [2], whose model was proposed by Parker [3] on the basis of his own and Biermann's [4-6] studies. Parker [3] opened a new era of research on the solar wind interaction with space bodies, in which the static picture of the Sun, with no solar particle emission, was replaced by the dynamic scenario. As a result of this research a plurality of phenomena associated with the existence of the solar wind were discovered. At this time, understanding of solar wind and its interaction with planets, comets and other bodies, became Rosetta stones of space physics $[7,8]$.

Mars is a planetary body that interacts with the solar wind in a peculiar way. In contrast to most of planets of the Solar System (Earth, Jupiter, Saturn, Neptune, or Uranus) that have an internal magnetic field, Mars has no global intrinsic magnetic field that would protect its gaseous environment. As a result of that, the solar wind interacts directly with the Martian environment. A bow shock formation, magnetic field draping, ion pick-up, mass-loading, boundary formation, ionopause formation and generation of the magnetotail, are examples of phenomena that compose the complex description of this interaction [9]. These theoretical findings of the solar wind interaction with Mars were verified by many spacecrafts which were sent towards the planet. Among others, the Soviet Mars-3 mission was successful in 1973 in finding a bow shock and a thick tail with a surrounding boundary layer containing traces of picked-up ions [10]. These findings were later confirmed by Phobos 2 [11].
A great impact on the Martian solar wind interaction studies was exerted by the Mars Global Surveyor mission (initiated in 1999). A large amount of data collected by this mission [12] stimulated a number of numerical simulations. For instance, Shinagawa [13] developed the two-dimensional (2D) magnetohydrodynamic model to analyse the electron mass density profile vs. the solar wind gas pressure [13]. He studied influence of the Martian crustal magnetic field by comparing the electron mass density profiles of Venus and Mars. On the other hand, Ma [14] showed in her multi-species model of the solar wind interaction with Mars that the crustal magnetic field exerts a small effect on the bow shock distortion. Shinagawa [15] described dynamics and structures of Martian and Venusian ionospheres controlled by the solar wind and reviewed the observations and theoretical studies of these ionospheres. A loss of planetary particles caused by the solar wind is also an important issue $[16,17]$. Ion escape fluxes from Mars were a subject of numerical studies in the frame of 3D multi-species model, in which different solar wind conditions resulting from the solar cycle were discussed [18].

Observational findings indicate that wave-like structures and instabilities arise at the Martian ionopause [19]. The goal of this paper is to study the ionopause waves and investigate generation of the Kelvin-Helmholtz (KH) and negative energy waves (NEW) instabilities, in order to describe the characteristic properties of plasma evolution at the ionopause.

This paper is structured as follows. In Sect. 2, our 2D hydrodynamic model of plasma is presented. In the following part of the draft, we derive a differential equation describing the evolution of surface gravity waves, which mimic the ionopause waves. The dispersion relation and analysis of the KH and NEW instabilities are presented in Sect. 4. Main results of this paper are summarized in Sect. 5.

\section{Physical model}

We consider the ionosphere of Mars in the 2D reference frame of which the $x$-axis is directed nightward along the 
ionopause and the $z$-axis points to the centre of Mars (Fig. 1). We assume that the system is invariant along the $y$-direction, $\partial / \partial y=0$, and the $y$-component of velocity is set to zero, $v_{y}=0$. We discuss a region around the terminator plane, in which the ionosheath plasma velocity is essentially parallel to the $x$-axis and treat the interface as a strictly definite border between two distinct environments, namely the ionosheath and ionospheric plasma. The uniform gravity field, $g=3 \mathrm{~m} \mathrm{~s}^{-2}$, is pointing inward the planet along the $z$-axis. We also assume that the plasma is magnetic field-free and incompressible $(\nabla \cdot \boldsymbol{v}=0)$. The former assumption is quite restrictive, but it gives a realistic approximation of plasma behaviour at the ionopause, where magnetic field is negligibly small [13]. The later assumption complies well with surface gravity waves which are essentially incompressible, e.g. Murawski [20].

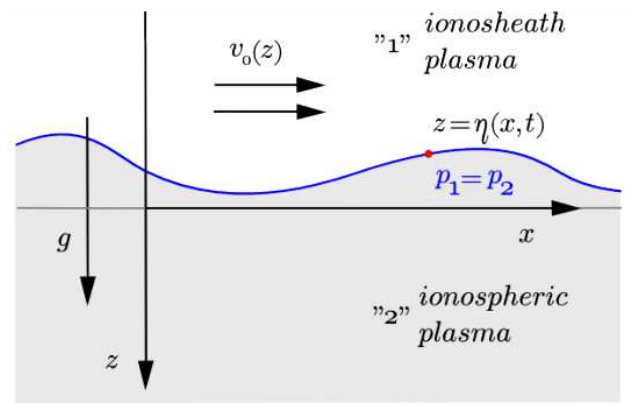

Fig. 1. Perturbed interface $z=\eta(x, t)$ between the ionosheath plasma and ionospheric plasma.

Although ionosheath plasma is penetrated by magnetic field the ionosphere of Mars is generally a magnetic-free medium. In the first approximation we can neglect magnetic field in the ionosheath and construct a model which would serve as a building block for development of more realistic models. A similar approach was made by Wang and Nielsen [21] who discussed the properties of hydrodynamic waves in the topside of ionospheres of Mars and Venus. They concluded that waves excited by solar wind fluctuation in the Martian topside ionosphere depend very weakly on magnetic field but waves are strongly influenced by the planetary gravitation. Assuming small velocity differences among electrons, ions and neutrals, Wang and Nielsen [21] obtained the hydrodynamic equations (Eq. (4) of Wang and Nielsen, [21]) governing the motion of the plasma. As a result, we focus on hydrodynamic aspects of plasma behaviour, leaving a magnetic field case for future studies. We believe that our model is suitable to the ionopause at the terminator region where a presence of essentially horizontal magnetic field can be neglected in a first approximation, for which our model is constructed.

Applying the above introduced assumptions, we model the ionopause waves by the incompressible Euler equations

$$
\begin{aligned}
& \frac{\partial \rho}{\partial t}+\boldsymbol{v} \cdot \nabla \rho=0, \quad \nabla \cdot \boldsymbol{v}=0, \\
& \rho \frac{\partial \boldsymbol{v}}{\partial t}+\rho(\boldsymbol{v} \cdot \nabla) \boldsymbol{v}=-\nabla p+\rho \boldsymbol{g}, \\
& \frac{\partial p}{\partial t}+\nabla(p \boldsymbol{v})=0 .
\end{aligned}
$$

Our model introduces two separate fluids demarcated by the interface, $z=0$ (Fig. 1). The ionosheath plasma, which flows with its speed $\boldsymbol{v}_{0}=v_{0}(z) \hat{x}$, occupies the region, $z<0$, and is denoted by index " 1 ". The ionosphere is present below the ionosheath plasma whose quantities are denoted by the index " 2 ".

The equilibrium gas pressure, $p_{0}$, varies with $z, p_{0}=$ $p_{0}(z)$, according to the hydrostatic condition,

$$
-\frac{\partial p_{0}}{\partial z}=g \rho_{0}
$$

while the equilibrium mass density, $\rho_{0}(z)$, is constant in the top and bottom media, experiencing a jump at $z=$ $\eta(x, t)$.

Boundary conditions at the perturbed interface, $z=$ $\eta(x, t)$, are established by setting

$$
p_{1}=p_{2}, \quad z=\eta(x, t) .
$$

Here $p_{1}\left(p_{2}\right)$ is evaluated in the top (bottom) medium. The assumption that the time rate of change of the surface in the frame of reference moving with the fluid with velocity $\boldsymbol{v}$ equals zero leads to

$$
\frac{\partial \zeta}{\partial t}+(\boldsymbol{v} \cdot \nabla) \zeta=0
$$

where

$$
\zeta=z-\eta(x, t)=0 .
$$

The above equation supplements the boundary condition (5) and allows only the motion of fluid along the perturbed interface.

\section{Wave equations for surface waves}

We consider small amplitude perturbations $\delta v_{x}, \delta v_{z}$, $\delta p$ around the equilibrium, such as

$$
\left\{\begin{array}{l}
\varrho=\varrho_{0}(z), \\
v_{x}=v_{0}(z)+\delta v_{x}(x, y, z), \\
v_{z}=0+\delta v_{z}(x, y, z), \\
p=p_{0}(z)+\delta p(x, y, z) .
\end{array}\right.
$$

Let us note that as a result of the incompressibility assumption mass density remains unperturbed. From Eq. (2) after dropping small terms and symbol $\delta$, we get

$$
\frac{\partial \boldsymbol{v}}{\partial t}+\left(\boldsymbol{v}_{0} \cdot \nabla\right) \boldsymbol{v}+(\boldsymbol{v} \cdot \nabla) \boldsymbol{v}_{0}=-\frac{1}{\varrho_{0}} \nabla p .
$$

Here $\boldsymbol{v}_{0}(z)=\left[v_{0}(z), 0\right]$. The $x$-component of this equation attains the form

$$
\frac{\partial v_{x}}{\partial t}+v_{0} \frac{\partial v_{x}}{\partial x}+v_{z} \frac{\partial v_{0}}{\partial z}=-\frac{1}{\varrho_{0}} \frac{\partial p}{\partial x} .
$$

Similarly, from the $z$-component we get

$$
\frac{\partial v_{z}}{\partial t}+v_{0} \frac{\partial v_{z}}{\partial x}=-\frac{1}{\varrho_{0}} \frac{\partial p}{\partial z} .
$$

As a result of the incompressibility condition, $\nabla \cdot \boldsymbol{v}=0$, 
the plasma can be described by the velocity flux function, $\Psi(x, y, z) \hat{y}$, such as

$$
\boldsymbol{v}=\nabla \times(\Psi \hat{y}) .
$$

In the flux function notation we rewrite Eqs. (9) and (10) as

$$
\begin{aligned}
& \frac{\partial^{2} \Psi}{\partial z \partial t}+v_{0} \frac{\partial^{2} \Psi}{\partial z \partial x}-\frac{\partial v_{0}}{\partial z} \frac{\partial \Psi}{\partial x}=-\frac{1}{\varrho_{0}} \frac{\partial p}{\partial x}, \\
& \frac{\partial^{2} \Psi}{\partial x \partial t}+v_{0} \frac{\partial^{2} \Psi}{\partial x^{2}}=\frac{1}{\varrho_{0}} \frac{\partial p}{\partial z} .
\end{aligned}
$$

Using the Taylor expansion

$$
f(z=\eta)=f(z=0)+\frac{\partial f}{\partial z}(z=0) \eta+\ldots
$$

in Eqs. (5) and (6), we obtain

$$
\begin{aligned}
& p_{1}+\frac{\partial p_{10}}{\partial z} \eta=p_{2}+\frac{\partial p_{20}}{\partial z} \eta, \quad z=0, \\
& \frac{\partial \eta}{\partial t}=-\frac{\partial \Psi}{\partial x}-v_{0} \frac{\partial \eta}{\partial x}, \quad z=0 .
\end{aligned}
$$

Equations (12) and (13) supplemented by the boundary conditions (15) and (16) consist the fundamental system for our problem. We consider now sinusoidal perturbations propagating along the $x$-direction,

$$
f(x, t) \sim \mathrm{e}^{\mathrm{i}(k x-\omega t)} .
$$

Here $f(x, t)$ corresponds to a perturbed quantity, $k$ is a wave number, and $\omega$ denotes the cyclic frequency.

Substituting Eq. (17) into Eqs. (9), (10), (15) and (16), we find

$$
\begin{aligned}
& \varrho_{0}\left(\frac{\omega}{k}-v_{0}\right) \frac{\partial \Psi}{\partial z}+\varrho_{0} \frac{\partial v_{0}}{\partial z} \Psi=p, \\
& \varrho_{0}\left(\frac{\omega}{k}-v_{0}\right) k^{2} \Psi=\frac{\partial p}{\partial z}, \\
& g\left(\varrho_{10}-\varrho_{20}\right) \eta=p_{2}-p_{1}, \quad z=0, \\
& \left(\frac{\omega}{k}-v_{0}\right) \eta=\Psi, \quad z=0 .
\end{aligned}
$$

Hence we obtain

$$
\begin{gathered}
g\left(\varrho_{10}-\varrho_{20}\right) \frac{1}{\left(\frac{\omega}{k}-v_{0}\right)} \Psi=\varrho_{20} \int \omega k \Psi_{2} \mathrm{~d} z \\
-\varrho_{10} \int\left(\frac{\omega}{k}-v_{0}\right) k^{2} \Psi_{1} \mathrm{~d} z, \quad z=0, \\
\Psi_{2}=\frac{\frac{\omega}{k}}{\frac{\omega}{k}-v_{0}} \Psi_{1}, \quad z=0 .
\end{gathered}
$$

Combining Eqs. (18) and (19), we get TaylorGoldstein equation [22]

$$
\frac{\partial^{2} \Psi}{\partial z^{2}}+\left[\frac{\frac{\partial^{2} v_{0}}{\partial z^{2}}}{\frac{\omega}{k}-v_{0}}-k^{2}\right] \Psi=0, \quad \frac{\omega}{k} \neq v_{0} .
$$

This equation describes the behaviour of small amplitude surface gravity waves which experience a resonant absorption at the altitude defined by the condition $\omega / k=$ $v_{0}(z)$.

\section{Dispersive curves for flowing plasma}

For simplicity reasons, we assume that the ionosheath plasma velocity is constant, $v_{0}(z)=$ const. Then, from Taylor-Goldstein Eq. (24) we get

$$
\Psi_{1}(z), \Psi_{2}(z) \sim \mathrm{e}^{-k z} .
$$

After substitution of the above expressions into Eqs. (22) and (23) we obtain the following quadratic dispersion relation in $\omega$ :

$$
\omega^{2}(\kappa+1)+\omega\left(-2 k v_{0}\right)+\left[k^{2} v_{0}^{2}-g k(1-\kappa)\right]=0 .
$$

Here the symbol $\kappa$ denotes the ratio of mass densities

$$
\kappa=\frac{\varrho_{10}}{\varrho_{20}} \text {. }
$$

We find two roots of Eq. (21)

$$
\omega_{ \pm}=\frac{k v_{0} \pm \sqrt{\left(1-\kappa^{2}\right) g k-\kappa v_{0}^{2} k^{2}}}{\kappa+1} .
$$

Here we consider $k$ to be real and positive, while $\omega$ can be imaginary with a positive (negative) real part corresponding to waves propagating into the positive (negative) $x$-direction. In the case of still medium $\left(v_{0}=0\right)$, we recover the result of Murawski [20], that is

$$
\omega_{ \pm}= \pm \frac{\sqrt{\left(1-\kappa^{2}\right) g k}}{\kappa+1}
$$

where $\omega_{+}\left(\omega_{-}\right)$corresponds to rightwardly (leftwardly) propagating waves. For $v_{0} \neq 0$ we study the influence of the ionosheath plasma flow on the wave period, $P=2 \pi /|\operatorname{Re}(\omega)|$, where $\operatorname{Re}(\omega)$ denotes a real part of $\omega$.
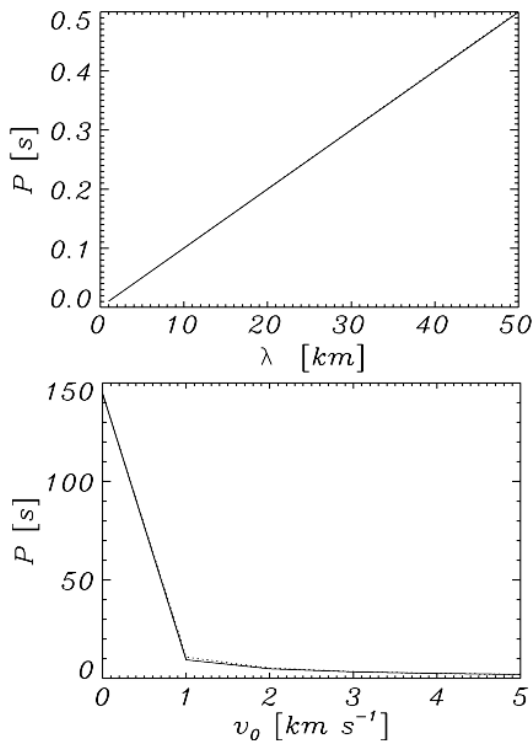

Fig. 2. Wave-period $P$ vs. the wavelength, $\lambda$, (top part) and vs. the flow velocity, $v_{0}$, (bottom part) corresponding to frequency $\omega_{+}$(solid line) and $\omega_{-}$ (dashed line) plotted with the use of Eq. (28) for $v_{0}=$ $100 \mathrm{~km} \mathrm{~s}^{-1}, \lambda=10 \mathrm{~km}$ and $\kappa=10^{-2}$.

We illustrate wave period, $P$, as a function of the wavelength, $\lambda=2 \pi / k$, for $v_{0}=100 \mathrm{~km} \mathrm{~s}^{-1}$ and $\kappa=10^{2}$ (Fig. 2, top part), which are typical ionospheric conditions [23]. As a result of the dominant Doppler term, $k v_{0} /(\kappa+1)$, in dispersion relation (28) $P$ attains similar values for $\omega_{+}$and $\omega_{-}$within the the range of chosen pa- 

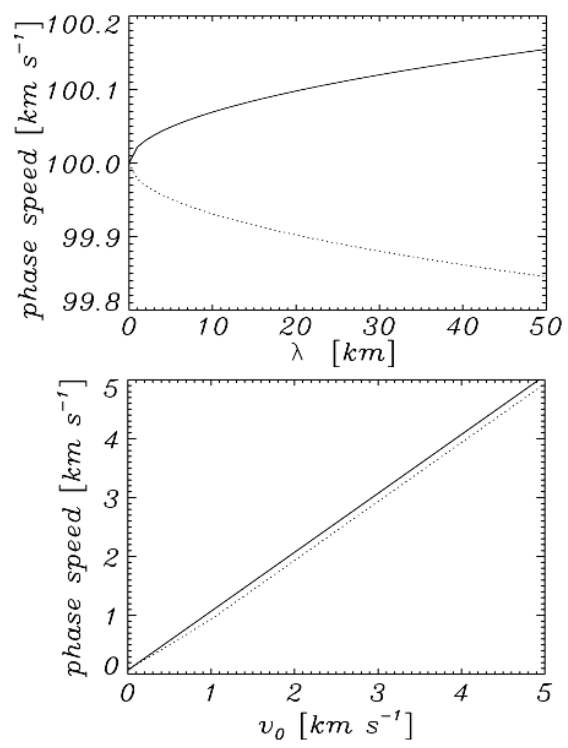

Fig. 3. Phase speed, $\omega / k$, resulting from Eq. (28) vs. $\lambda$ (top part) and vs. the horizontal flow velocity, $v_{0}$, (bottom part) for $\omega_{+}$(solid line) and $\omega_{-}$(dashed line), with $v_{0}=100 \mathrm{~km} \mathrm{~s}^{-1}, \lambda=10 \mathrm{~km}$ and $\kappa=10^{-2}$.

rameters. Small dispatches in $P$ between $\omega_{+}$and $\omega_{-}$are discernible for long wavelength waves.

Figure 2 (bottom part) shows the dependence of $P$ on $v_{0}$. Notice that wave periods corresponding to $\omega_{+}$and $\omega_{-}$do not differ significantly. It can be seen that wave period for both frequencies $\omega_{+}$and $\omega_{-}$decreases with flow velocity and falls off to zero in the limit $v_{0} \rightarrow \infty$. Phase speed $\omega / k$ experiences modifications for different values of $\lambda$ (Fig. 3, top part), and $v_{0}$ (Fig. 3, bottom part). In Fig. 3 (top part), both phase speeds for $\omega_{+}$and $\omega_{-}$reveal symmetry towards given flow velocity $v_{0}=100 \mathrm{~km} \mathrm{~s}^{-1}$, while in Fig. 3 (bottom part), values of the phase speeds are slightly shifted with respect to each other above $v_{0}=1 \mathrm{~km} \mathrm{~s}^{-1}$.

We now study the dispersion of surface waves at the ionopause. The wave dispersion is defined as:

$$
\frac{\partial^{2} \omega}{\partial k^{2}}=\frac{-\left[\left(1-\kappa^{2}\right) g\right]^{2}}{4(\kappa+1)\left[\left(1-\kappa^{2}\right) g k-\kappa v_{0}^{2} k^{2}\right]^{\frac{3}{2}}} .
$$

As $k$ approaches zero, the dispersion tends to infinity

$$
\lim _{k \rightarrow 0} \frac{\partial^{2} \omega}{\partial k^{2}} \longrightarrow \infty \text {. }
$$

Hence, we infer that infinity long waves are infinitely dispersive. On the other hand, the dispersion tends to zero, while the wave number approaches infinity

$$
\lim _{k \rightarrow \infty} \frac{\partial^{2} \omega}{\partial k^{2}} \longrightarrow 0 \text {. }
$$

So, infinitely short waves are non-dispersive. The wave dispersion is plotted with the use of Eq. (30) vs. wavelength, $\lambda$, in Fig. 4 (top part). We infer that short wavelength surface waves propagating along the Martian ionopause are less dispersive than longer waves. These
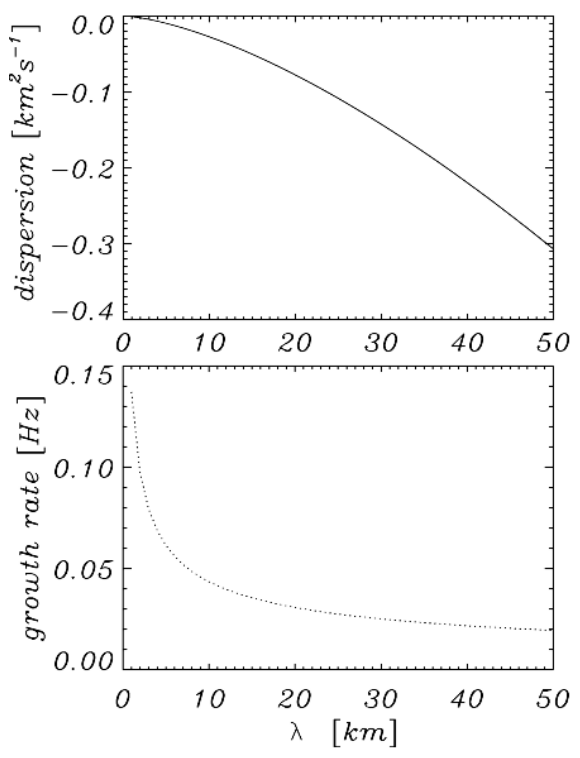

Fig. 4. Dispersion, $\partial^{2} \omega / \partial k^{2}$, vs. $\lambda$ plotted with the use of Eq. (30) for $\omega_{+}$(solid line) and $\omega_{-}$(dashed line) (top part) and the variation of a growth rate $\omega_{i}$ of unstable Kelvin-Helmholtz mode vs. $\lambda$ (bottom part).

numerical findings remain in agreement with the analytical results of Eqs. (31) and (32).

\subsection{Kelvin-Helmholtz instabilities}

Kelvin-Helmholtz instabilities (KHI) occur for a sufficiently large gradient flow [24]. In a Martian case, we have gradient in velocity between ionosheath and ionospheric plasma. Occurrence of KHI is an important phenomenon, because it contributes to loss of planetary ions [25]. Penz et al. [17] showed that atmospheric ions loss is caused by KHI occurring at equatorial flanks of the terminator plane. For these instabilities, the term under the square root in Eq. (28) must be negative [26],

$$
\left(1-\kappa^{2}\right) g k-\kappa v_{0}^{2} k^{2}<0 .
$$

As a result of that $\omega$ attains a non-zero imaginary part $\omega_{i}=\operatorname{Im}\left(\omega_{i}\right)$. From Eq. (33) it follows that the KHI are present if the flow exceeds the threshold

$$
v_{\mathrm{cKH}}=\sqrt{\frac{\left(1-\kappa^{2}\right) g}{\kappa k}} .
$$

Figure 5 (top part) illustrates the critical velocity for KHI, $v_{\mathrm{cKH}}$, as a function of $\lambda$. The critical velocity for KHI grows with wavelength for $\kappa=10^{-2}$. This kind of instabilities results in vortices and consequently in turbulence as well as mixing of flow at the interface.

Variation of growth rate $\omega_{i}$ of unstable KelvinHelmholtz mode with wavelength $\lambda$ is shown in Fig. 4 (bottom part). Let us notice that as $\omega_{i}$ declines with $\lambda$ we infer that short wavelength waves are more prone for $\mathrm{KH}$ instabilities than longer ones. Detailed study showing variation of growth rate depending on parameters like mass density ratio and velocity was investigated by 


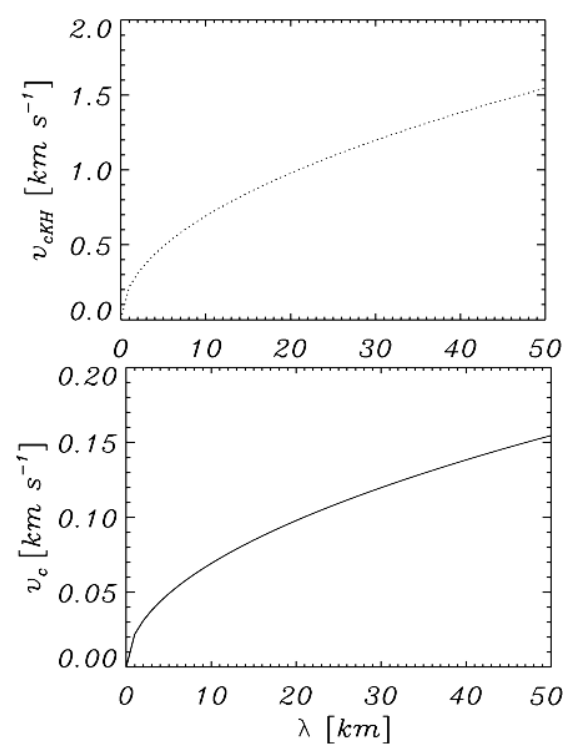

Fig. 5. Critical velocity for KHI (top part) and NEW (bottom part) vs. $\lambda$ for $\kappa=10^{-2}$.

Amerstorfer et al. [27, 28] depending on plasma parameters.

\subsection{Negative energy waves instabilities}

The negative energy waves (NEW) are associated with the presence of backward waves [24]. Amplitude of NEW grows, while the total energy of the system is decreased. This growth results from dissipative effects, while the system tries to reduce its energy and thus leading to amplitude growth. Because of energy transfer from the steady flow to NEW, the law of the energy conservation is satisfied [24]. To determine whether waves are NEW, we use the criterion introduced by Cairns [29], i.e.

$$
C_{ \pm}=\omega \frac{\partial D_{ \pm}}{\partial \omega}<0 .
$$

Here, the symbol $D_{ \pm}$denotes the dispersion relation, which is multiplied by $a_{ \pm}= \pm 1$. A value of $a_{+}$has to be determined from the condition $C_{+}>0$ for $v_{0}=0$ [24]. As a result for $\omega=\omega_{+}\left(\omega=\omega_{-}\right)$we have $a_{+}=1\left(a_{-}=-1\right)$. For our problem it can be expressed in the following form:

$$
D_{ \pm} \equiv a_{ \pm}\left[\omega^{2}(\kappa+1)-2 k v_{0} \omega+k^{2} v_{0}^{2}-g k(1-\kappa)\right] .
$$

If the value of $C_{ \pm}$is negative, waves are NEW. We find

$$
C_{ \pm}=2 \omega_{ \pm} a_{ \pm}\left[\omega_{ \pm}(\kappa+1)-k v_{0}\right] \text {. }
$$

We consider first the positive frequency, $\omega_{+}$, of Eq. (28) for that the Cairns condition becomes

$$
\begin{aligned}
C_{+} & =\frac{2}{\kappa+1}\left[\sqrt{\left(1-\kappa^{2}\right) g k-\kappa v_{0}^{2} k^{2}}+k v_{0}\right] \\
& \times \sqrt{\left(1-\kappa^{2}\right) g k-\kappa v_{0}^{2} k^{2}}<0 .
\end{aligned}
$$

Usually NEW are present for flow velocities $v_{0}$ lower than the Kelvin-Helmholtz threshold velocity [24]. It means that square root in Eq. (38) must be positive

$$
\sqrt{\left(1-\kappa^{2}\right) g k-\kappa v_{0}^{2} k^{2}}>0
$$

and the Cairns condition gives then

$$
\sqrt{\left(1-\kappa^{2}\right) g k-\kappa v_{0}^{2} k^{2}}<-k v_{0} .
$$

As this equation is never satisfied for $k>0$ and $v_{0}>$ 0 we infer that there are no NEW for the rightwardly propagating waves.

On the other hand, for the leftwardly propagating waves with $\omega_{-}$, given by Eqs. (28)-(35), we have

$$
\begin{aligned}
C_{-} & =\frac{2}{\kappa+1}\left[\sqrt{\left(1-\kappa^{2}\right) g k-\kappa v_{0}^{2} k^{2}}-k v_{0}\right] \\
& \times \sqrt{\left(1-\kappa^{2}\right) g k-\kappa v_{0}^{2} k^{2}}<0 .
\end{aligned}
$$

Both solutions of Eq. (28) in form of Eq. (38) and (41) are presented in Fig. 6 (top part). It is discernible that only solution of Eq. (28) denoted as $\omega_{-}$gives NEW (Fig.6, bottom part). As a result, only backward propagating waves for a sufficiently large value of flow $v_{0}$ become NEW (Fig.6, top part).
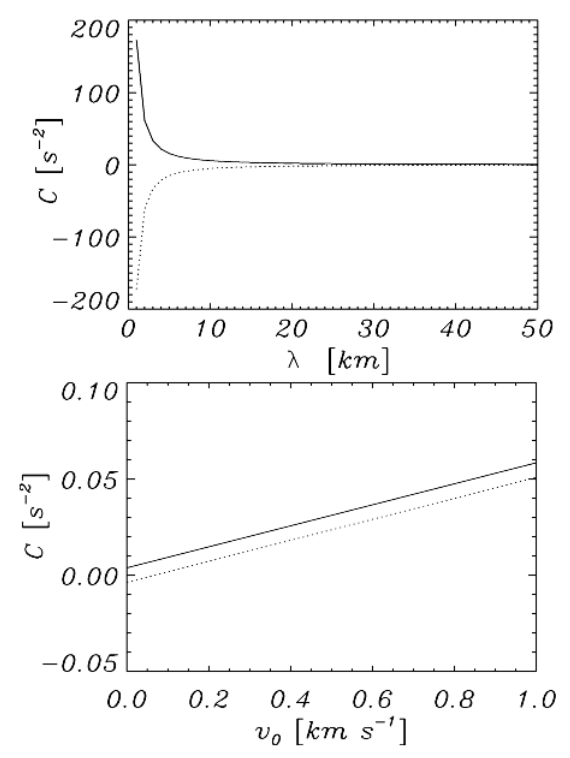

Fig. 6. Cairns criterion for positive and negative frequencies, $\omega_{+}$(solid line) and $\omega_{-}$(dashed line), vs. horizontal wave number, $k$ for flow velocity $v_{0}=100 \mathrm{~km} \mathrm{~s}^{-1}$ (top part) and flow velocity, $v_{0}$ for $\lambda=10 \mathrm{~km}$ (bottom part).

From Eq. (28) or (41) we can derive a critical velocity

$$
v_{\mathrm{c}}=\sqrt{\frac{(1-\kappa) g}{k}} \text {. }
$$

The backward propagating waves become NEW for values of the ionosheath velocity higher than $v_{\mathrm{c}}$. Variation of critical velocity for NEW, $v_{\mathrm{c}}$, with wavelength, $\lambda$, is displayed in Fig. 5 (bottom part), which reveals that $v_{\mathrm{c}}$ increases with $\lambda$.

\section{Summary}

In this paper we discussed the problem of the surface wave propagation along the ionopause of Mars and de- 
rived the conditions under which the Kelvin-Helmholtz instabilities and negative energy waves arise due to ionosheath plasma flow. In the two-dimensional model that we originally developed to describe a perturbed interface, we considered the region around the terminator plane. We implied such equilibrium for which the ionosheath medium flows parallel to the ionopause but fluid remains still $(\boldsymbol{v}=\mathbf{0})$ below the interface. For such conditions, we obtained the dispersion relation for surface waves, which was illustrated for the realistic set of fluid parameters. We estimated wave period, phase speed, dispersion and critical flow velocities which result in instabilities seeded by ionosheath plasma flow. Our investigation of the dispersion relation showed that waves in this region are weakly dispersive and they are unstable for sufficiently short wavelengths. Our analysis leads to a conclusion that the Kelvin-Helmholtz instabilities and negative energy waves could occur even for relatively low flow velocities.

\section{Acknowledgments}

N.K. is thankful to the Institute of Physics, UMCS, Lublin for providing the financial support of his scientific visit to UMCS.

\section{References}

[1] W. Sheehan, The Planet Mars: A History of Observation and Discovery, University of Arizona Press, Tucson 1996

[2] K.I. Gringauz, V.G. Kurt, V.I. Moroz, I.S. Shklovskii, Astronomich. Zh. 37, 716 (1960).

[3] E.N. Parker, Astrophys. J. 128, 664 (1958).

[4] L. Biermann, Zeits. Astrophys. 29, 274 (1951).

[5] L. Biermann, Zeits. Naturforsch. Teil A 7, 127 (1952).

[6] L. Biermann, Observatory 77, 109 (1957).

[7] C.T. Russell, Am. Geophys. Union, Space Weather, Geophys. Monogr. 125, 73 (2001).

[8] E.N. Parker, Space Sci. Rev. 9, 325 (1969).

[9] J.G. Luhmann, Space Sci. Rev. 44, 241 (1986).
[10] O.L. Vaisberg, Adv. Space Res. 12, 311 (1976).

[11] Ye. Yeroshenko, W. Riedler, K. Schwingenschuh, G.J. Luhmann, M. Ong, C.T. Russell, Geophys. Res. Lett. 17/6, 885 (1990).

[12] D.A. Brain, Space Sci. Rev. 126, 77 (2006).

[13] H. Shinagawa, S.W. Bougher, Earth Planets Space 51, 55 (1999).

[14] Y.J. Ma, A.F. Nagy, K.C. Hansen, D.L. DeZeeuw, T.I. Gombosi, K.G. Powell, J. Geophys. Res. 107, 1282 (2002)

[15] H. Shinagawa, Adv. Space Res. 33, 1924 (2004).

[16] S.H. Brecht, S.A. Ledvina, Space Sci. Rev. 126, 15 (2006).

[17] T. Penz, N.V. Erkaev, H.K. Biernat, H. Lammer, U.V. Amerstorfer, H. Gunell, E. Kallio, S. Barabash, S. Orsini, A. Milillo, W. Baumjohann, Planetary Space Sci. 52, 1157 (2004).

[18] Y.J. Ma, A.F. Nagy, Geophys. Res. Lett. 34, 8201 (2007).

[19] H. Gunell, U.V. Amerstorfer, H. Nilsson, C. Grima, M. Koepke, M. Fränz, J.D. Winningham, R.A. Frahm, J.A. Sauvaud, A. Fedorov, N.V. Erkaev, H.K. Biernat, M. Holmström, R. Lundin, S. Barabash, Plasma Phys. Control. Fusion 50, 074018 (2008).

[20] K. Murawski, Astrophys. J. 537, 495 (2000).

[21] J.S. Wang, E. Nielsen, J. Geophys. Res. 107, 1039 (2002).

[22] P.H. LeBond, L.A. Mysak, Waves in the Ocean, Elsevier Oceanography Series, Elsevier, Amsterdam 1978.

[23] Y.J. Ma, A.F. Nagy, K.C. Hansen, I.V. Sokolov, J. Geophys. Res. 109, A07211 (2004)

[24] P.S. Joarder, V.M. Nakariakov, B. Roberts, Solar Phys. 176, 285 (1997).

[25] U.V. Möstl, N.V. Erkaev, M. Zellinger, H. Lammer, H. Gröller, H.K. Biernat, D. Korovinskiy, Icarus 216, 476 (2011)

[26] C.N. Lashmore-Davies, Plasma Phys. 71, 101 (2005)

[27] U.V. Amerstorfer, N.V. Erkaev, D. Langmayr, H.K. Biernat, Planetary Space Sci. 55, 1811 (2007)

[28] U.V. Amerstorfer, H. Gunell, N.V. Erkaev, H.K. Biernat, Astrophys. Space Sci. Transact. 5, 39 (2009).

[29] R.A. Cairns, J. Fluid Mech. 92, 1 (1979) 